\title{
Fabrication of Nickel Oxide and Ni-doped Indium Tin Oxide Thin Films
}

\section{Using Pyrosol Process}

Akihiko Nakasa ${ }^{1}$, Mami Adachi ${ }^{1}$, Hisanao Usami ${ }^{1}$, Eiji Suzuki ${ }^{1}{ }^{*}$, and Yoshio Taniguchi ${ }^{2}$

${ }^{1}$ Department of Fine Material Engineering, ${ }^{2}$ Department of Functional Polymer Science, Faculty of Textile

Science \& Technology, Shinshu University, 3-15-1, Tokida, Ueda-shi, Nagano, 386-8567, Japan

*Correspondent: esuzuki@giptc.shinshu-u.ac.jp 


\begin{abstract}
Organic light emitting diodes (OLEDs) need indium tin oxide (ITO) anodes with highly smooth surface. The work function of ITO, about $4.8 \mathrm{eV}$, is generally rather lower than the optimum level for application to OLEDs. In this work, $\mathrm{NiO}$ was deposited by pyrosol process on pyrosol ITO film to increase the work function of the ITO for improving the performance of OLEDs. It was confirmed that $\mathrm{NiO}$ was successfully deposited on pyrosol ITO film and the $\mathrm{NiO}$ deposition increased the work function of pyrosol ITO, using x-ray diffraction (XRD), field emission scanning electron microscopy (FE-SEM), atomic force microscopy (AFM) and atmospheric photoelectron spectroscopy. Furthermore, doping ITO with Ni succeeded in producing the Ni-doped ITO film with high work function and lower sheet resistance.
\end{abstract}

Keywords: nickel oxide; pyrosol process; work function 


\section{Introduction}

ITO has been widely used, because of its transparency in the visible light region and high conductivity, as an electrode for several electronic devices, such as OLEDs [1], liquid crystal displays [2], and solar cells [3]. Various methods have been reported for preparing ITO, such as sputtering [4], chemical vapor deposition [5], electron beam deposition [6], and spray pyrolysis [7-10].

Among the methods listed above, the spray pyrolysis method, called 'pyrosol method' in this report, is unique because it is applied at atmospheric pressure. The method is based on the pyrolysis of an aerosol produced by ultrasonic spraying. For commercial production of ITO, the pyrosol process requires less initial investment and operational expense, because of its atmospheric pressure operation, in comparison with other methods conducted at a high vacuum.

The work function of pyrosol ITO is usually lower, by approximately $1 \mathrm{eV}$, than the highest occupied molecular orbital (HOMO) of the organic layer of OLEDs [12]. The larger energy difference between the ITO and the organic layer makes the interfacial Schottky barrier larger, which results in a smaller injected current at the interface. Raising the work function of pyrosol ITO to a level close to the HOMO of the organic layer, therefore, would minimize the Schottky barrier, and resultantly enhance hole injection from the ITO to the organic layer of OLED, reducing the required voltage of the OLED.

Several methods, such as plasma treatments [13], UV treatments [14, 15] and grafting of molecules [16], have been reported for treating ITO, in an effort to increase its work function. Among them, the oxygen plasma treatment is known as the most effective technique in increasing the work function of ITO prepared by sputtering [13].

In this study, we prepared $\mathrm{NiO}$ thin film on ITO with high work function and hole transfer and injection properties using the pyrosol method. Furthermore, by doping ITO with nickel, Ni-doped ITO was produced with the high work function and low sheet resistance. Although the NiO film and the Ni-doped ITO film with high work function prepared by sputtering have been reported [17, 18], we will report that these materials can be prepared by the industrially favorable pyrosol method. 


\section{Experimental Details}

The details of the pyrosol method have been previously reported [7-10]. The reactant solution was atomized by ultrasonic vibration at $818 \mathrm{kHz}$ and carried by $5 \mathrm{~L} / \mathrm{min}$ air, flowing through a $50-\mathrm{mm}$ diameter tube, to the surface of the substrate $(50 \mathrm{~mm} \times 50 \mathrm{~mm})$ placed on an electric heater. The temperature of the substrate was controlled at a $500^{\circ} \mathrm{C}$, by adjusting the power input to the heater. The deposition time was 5 minutes.

The reactant solution used for the fabrication of $\mathrm{NiO}$ thin films by pyrosol process was prepared according to the following step. Iso- $\mathrm{PrOH}$, which was used as the solvent in this study, was added to $\mathrm{Ni}(\mathrm{OAc})_{2}$. By dropwisly adding of diethanolamine (DEA) of twice moles of $\mathrm{Ni}, \mathrm{Ni}(\mathrm{OAc})_{2}$ became soluble in the Iso-PrOH. 0.2 $\mathrm{M} \mathrm{Ni}(\mathrm{OAc})_{2}$ solution was used in this study.

The surface morphology and the structural properties of the ITO films were examined by atomic force microscopy (AFM) (Seiko Instruments SPA 400), x-ray diffraction (XRD) (Rigaku, CN4148B2) with Cu Ka radiation $(40 \mathrm{kV}, 30 \mathrm{~mA})$, and field emission scanning electron microscopy (FE-SEM) (Hitachi Co. Ltd. $\mathrm{S}-5000)$. AFM was performed in the contact mode using triangular $\mathrm{Si}_{3} \mathrm{~N}_{4}$ cantilevers in air, at room temperature. The surface work function was determined with an atmospheric photoelectron spectroscope (Riken Keiki, AC-2). The sheet resistance of the ITO substrates was measured using a four-point probe resistance meter (Mitsubishi Yuka, Lorester IP MCP-T250). 


\section{Results and Discussion}

3.1. Characterization of as-deposited pyrosol NiO on ITO

\subsubsection{Morphology and crystal structure}

First, it was confirmed that morphology of ITO film and NiO film deposited on ITO film using FE-SEM. The result is shown in Fig. 1. As seen from these figures, the grains of ITO is larger than those of the NiO film on ITO and it turns out that the NiO film deposited on ITO consists of about $50 \mathrm{~nm}$ uniform grains although ITO film consists of about $200 \mathrm{~nm}$ grains. The $\mathrm{NiO}$ deposition made the ITO surface smoother. Since uneven surface of electrode induces short-circuit in OLEDs operation, this $\mathrm{NiO}$ deposition is favorable to suppress short-circuit. Then, the roughness of film was examined by AFM measurement, and Ra and Rp-v values of the NiO film deposited on glass are shown in Figure 2. Both Ra and Rp-v values were decreased as deposition time increased. At $20 \mathrm{~min}$. deposition time, Ra and Rp-v were $1.8 \mathrm{~nm}$ and $21.0 \mathrm{~nm}$, respectively. Formation of $\mathrm{NiO}$ crystal film was confirmed by XRD analysis using the JCPDS card [19].

\subsubsection{Conductivity}

Then, sheet resistance of the $\mathrm{NiO}$ film deposited on ITO was plotted to deposition time in Fig. 3. It turns out that sheet resistance tends to increase with the increase in deposition time presumably because NiO film becomes thicker.

\subsubsection{Work function}

Furthermore, the work functions of the NiO films on glass and on ITO were plotted to deposition time in Fig. 4. As Ra and sheet resistance did, the work function also changed depending on deposition time. It increased with deposition time. The work function of $\mathrm{NiO}$ film deposited on ITO was especially high $5.4 \mathrm{eV}$. The difference between the work function of the $\mathrm{NiO}$ film deposited on glass and that of the $\mathrm{NiO}$ on ITO became conspicuous as deposition time was extended. This difference is thought to be due to crystal growth and interface band bending that vary depending on substrates.

\subsection{Characterization of Ni-doped pyrosol ITO}

Although the work function of ITO was successfully raised by depositing $\mathrm{NiO}$ on it, the sheet resistance increased, which is not favorable for OLED application. We tried, therefore, co-deposition of the precursor of $\mathrm{NiO}(15 \mathrm{ml})$ and that of ITO $(0.2 \mathrm{M}$ indium acetylacetonate (III) and $0.01 \mathrm{M}$ di-n-butyl tin diacetate in acetyl acetone, $135 \mathrm{ml}$ ). The work function of the co-deposition film, that is, Ni-doped ITO film is shown in Fig. 5. The work function of co-deposition film was as high as that of NiO deposited on ITO. Moreover, the sheet resistance of Ni-doped ITO was remarkably low; it was $\sim 40 \Omega / \square$ on glass substrate, and $\sim 10 \Omega / \square$ on ITO substrate. 


\section{Conclusion}

ITO was modified with depositing thin $\mathrm{NiO}$ film on its surface by the pyrosol method. The surface smoothness was improved and the work function was raised by NiO deposition, which is favorable for OLED electrode application. The sheet resistance, however, increased. Co-deposition of Ni and ITO thin film on ITO substrate by the pyrosol method modified ITO to be of a high work function and a low sheet resistance. Thus versatility of the pyrosol method, a simple method operated at atmospheric pressure was demonstrated.

\section{Acknowledgements}

This work was supported by the Cooperative Link for Unique Science and Technology for Economy Revitalization (CLUSTER) of Japan's Ministry of Education, Culture, Sports, Science and Technology. Dr. A. Nakasa was supported by 21 century COE program. 


\section{References}

[1] C. W. Tang, S. A. Van Slyke, Appl. Phys. Lett. 51 (1987) 913.

[2] M. Monkade, Z. Fuliang, P. Martinot-Lagarde, G. Durand, Appl. Phys. Lett. 50 (1987) 856.

[3] C. W. Tang, Appl. Phys. Lett. 48 (1986) 183.

[4] J. C. C. Fan, J. B. Goodenough, J. Electron. Soc. 122 (1975) 1719.

[5] R. G. Liversey, E. Lyford, H. Moore, J. Phys. E 1 (1968) 947.

[6]I. Hamberg, C. G. Granqvist, J. Appl. Phys. 60 (1986) 11.

[7] G. Blandenet, M. Court, Y. Lagarde, Thin Solid Films 77 (1981) 81.

[8] R. Pommier, C. Grill, J. Marucchi, Thin Solid Films 77 (1981) 91.

[9] Z. B. Zhou, R. Q. Cui, Q. J. Pang, Y. D. Wang, F. Y. Meng, T. T. Sun, Z. M. Ding, X. B. Yu, Appl. Surf. Sci. $172(2001) 245$.

[10] K. Maki, N. Komiya, A. Suzuki, Thin Solid Films 445 (2003) 224.

[11] N. G. Park, M. Y. Kwak, B. O. Kim, O. K. Kwon, Y. K. Kim, Y. S. Kim, Jpn. J. Appl. Phys. 41 (2002) 1523.

[12] C. Adachi, Y. Nakajima, T. Oyamada, Data book on work function of organic thin films (2003).

[13] K. H. Lee, H. W. Jang, K. -B. Kim, Y. -H. Tak, J. -L. Lee, J. Appl. Phys. 95 (2004) 586.

[14] M. G. Mason, L. S. Hung, C. W. Tang, S. T. Lee, K.W. Wong, M. Wang, J. Appl. Phys. 86 (1999) 1688.

[15] K. Sugiyama, H. Ishi, Y. Ouchi, K. Seki, J. Appl. Phys. 87 (2000) 295.

[16] C. Ganzorig, K. Kwak, K. Yagi, M. Fujihira, Appl. Phys. Lett. 79 (2001) 272.

[17] I. -M. Chan, T. -Y. Hsu, F. C. Hong, Appl. Phys. Lett., 81 (2002) 1899.

[18] C. -M. Hsua, W. -T. Wu, Appl. Phys. Lett. 85 (2004) 840.

[19] JCPDS card No. 441159. 


\section{Figure captions}

Fig. 1. The FE-SEM images of ITO deposited on glass by the pyrosol process.

Fig. 2. The Ra and Rp-v surface roughness parameters of NiO film on ITO, deposited on glass by the pyrosol process, at various deposition time $(\bullet$ : Ra, $\Delta$ : Rp-v).

Fig. 3. Resistance of as-deposited pyrosol $\mathrm{NiO}$ on ITO vs. deposition time.

Fig. 4. Work function of as-deposited pyrosol $\mathrm{NiO}$ on ITO, and $\mathrm{NiO}$ on glass vs. deposition time. (•: on ITO, $\square$ : on glass).

Fig. 5. Work function of as-deposited pyrosol Ni doped ITO obtained from photoelectron spectrospoy.. 

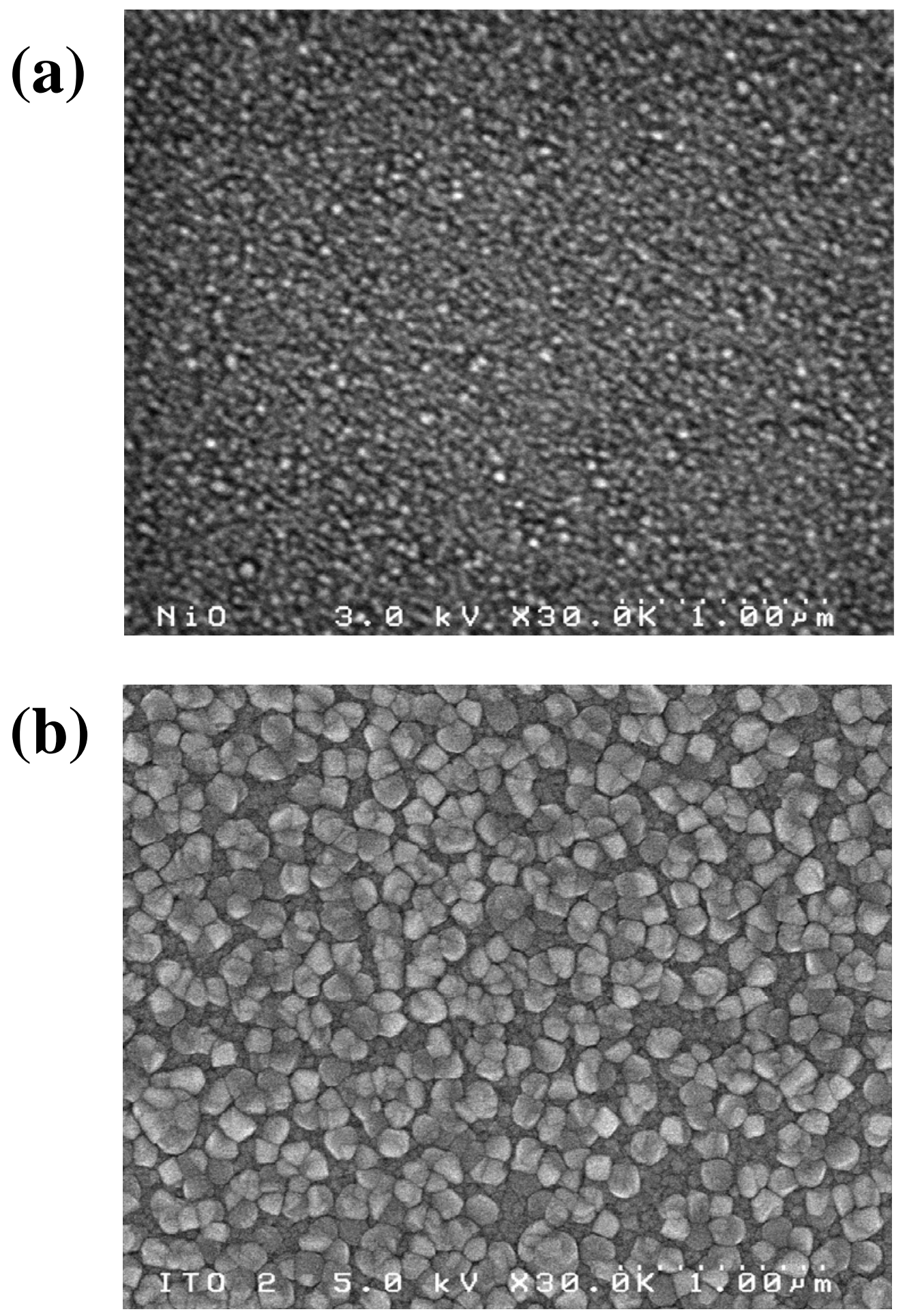

\section{Fig.1}




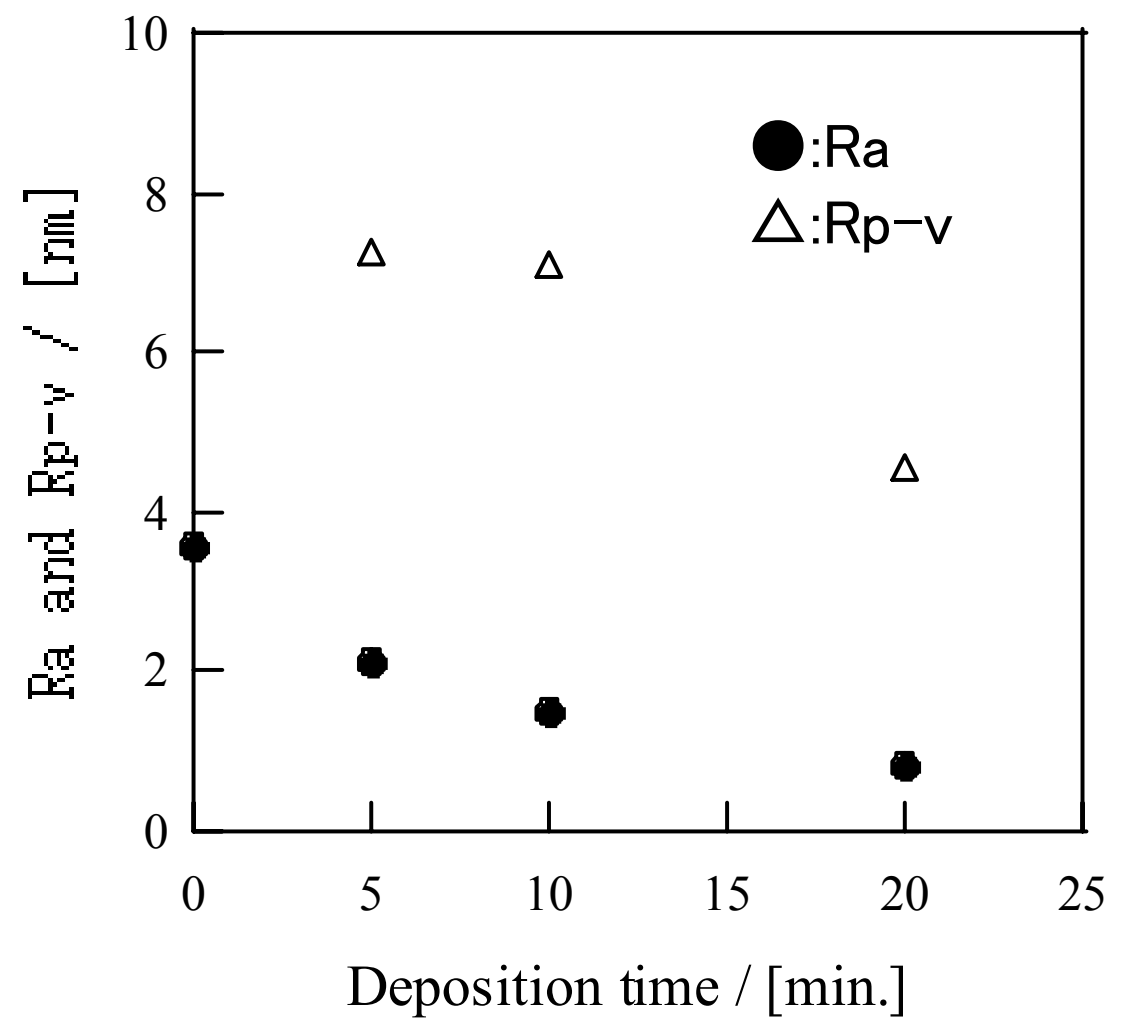

Fig.2 


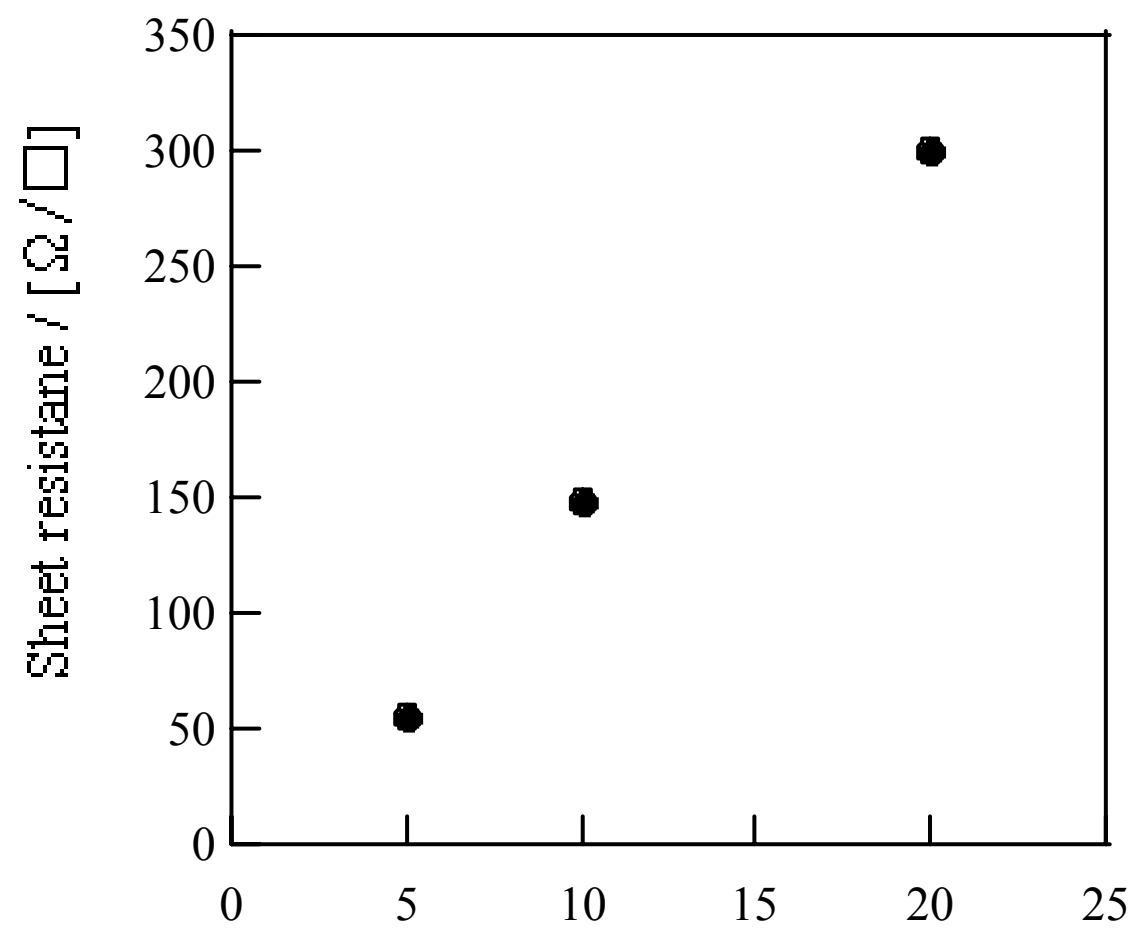

Deposition time / [min.] 


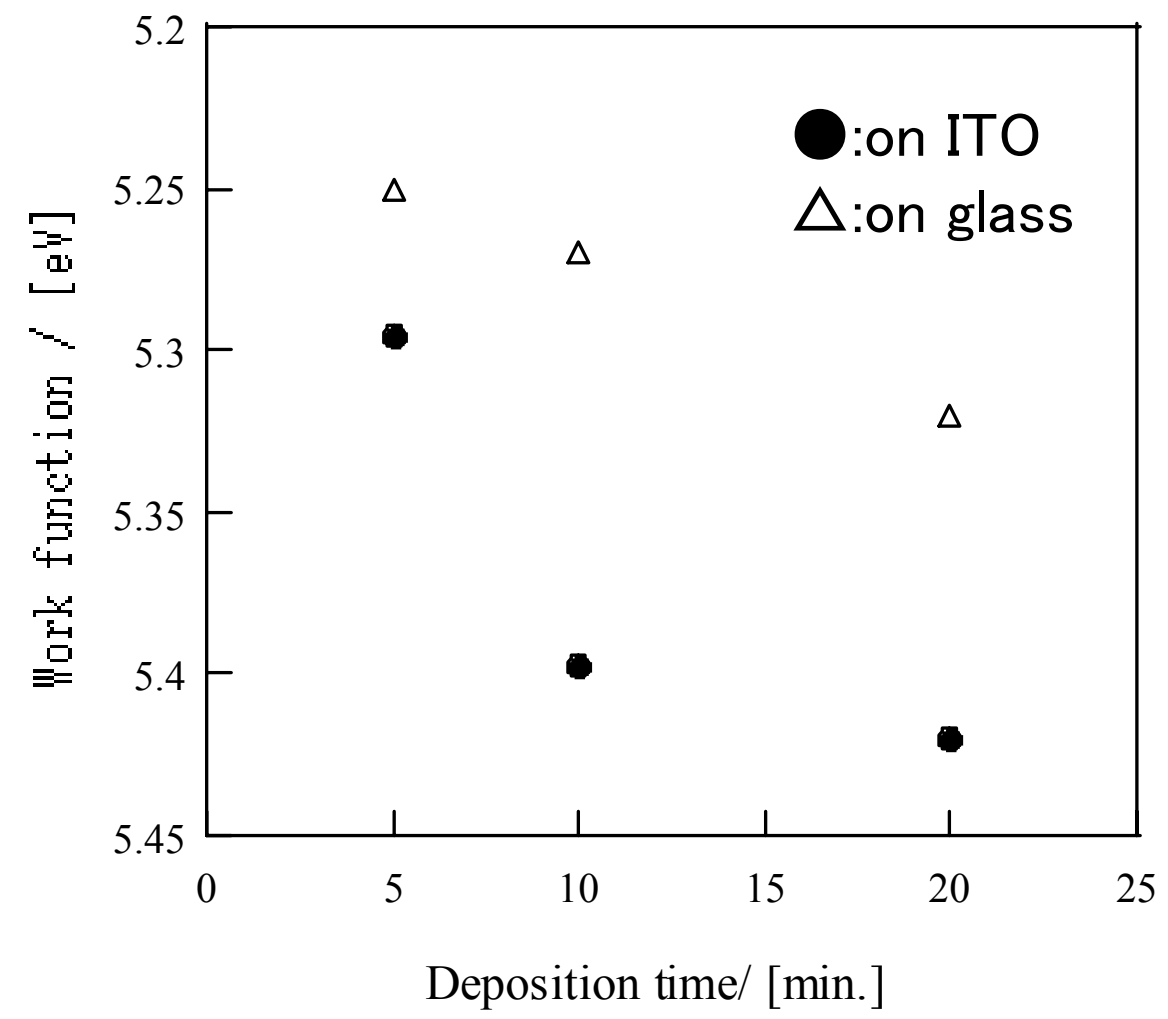

Fig.4 


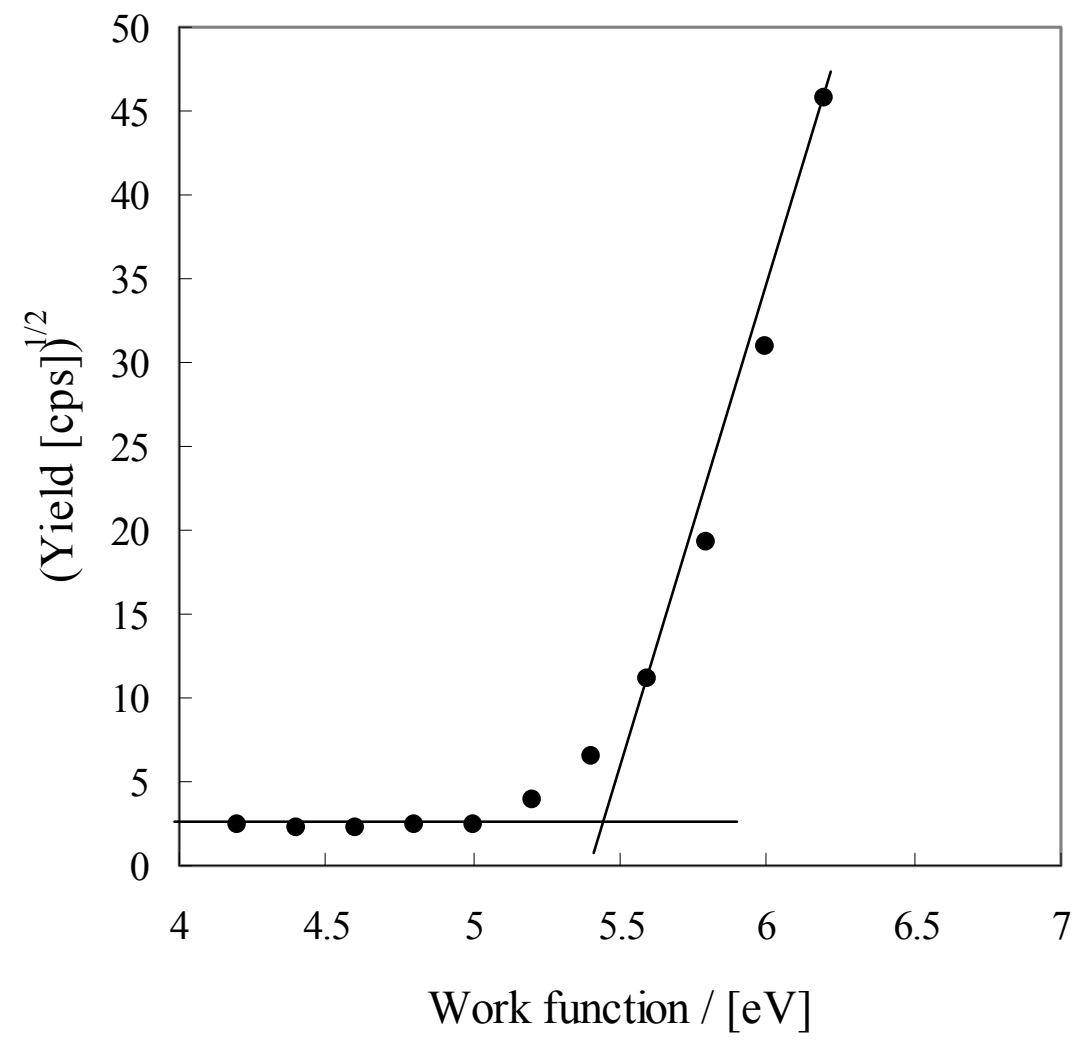

Fig.5 